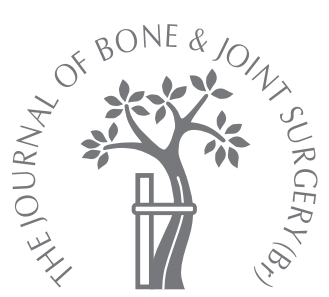

C. Y. Chung,

M. S. Park,

I. H. Choi,

T.-J. Cho,

W. J. Yoo,

K. M. Lee

From Seoul National

University, Seoul,

Korea

\title{
Morphometric analysis of acetabular dysplasia in cerebral palsy
}

We carried out a morphometric analysis of acetabular dysplasia in patients with cerebral palsy requiring hip surgery using three-dimensional CT. We evaluated 54 hips in 27 patients. The contralateral normal hips of ten patients with unilateral Perthes' disease were used as a control group. The acetabular defects were assessed qualitatively as anterior, posterior or global. Quantitative assessments were made using three-directional acetabular indices (anterosuperior, superolateral and posterosuperior) and measured by multiplanar reformation, from which we calculated the acetabular volume.

In the qualitative study, posterior defects were most common in the subluxation group whereas global defects predominated in the dislocation group. In the quantitative study, all acetabular indices in both the subluxation and dislocation groups were higher than those in the control group and the superolateral indices showed a tendency to elevation in the dislocation group. The acetabular volume was largest in the control group, smallest in the dislocation group, and intermediate between the two in the subluxation group.

C. Y. Chung, MD, Orthopaedic Surgeon, Professor

M. S. Park, MD,

Orthopaedic Surgeon,

Fellow

Department of Orthopaedic Surgery

Seoul National University

Bundang Hospital, 300

Gumi-Dong, Bundang-Gu,

Sungnam, Kyungki 463-707,

Korea.

I. H. Choi, MD, Orthopaedic Surgeon, Professor

- T.-J. Cho, MD, Orthopaedic

Surgeon, Associate

Professor

W. J. Yoo, MD,

Orthopaedic Surgeon,

Assistant Professor

K. M. Lee, MD,

Orthopaedic Surgeon,

Fellow

Department of Orthopaedic

Surgery

Seoul National University

Children's Hospital, 28

Yongun-Dong, Chonro-Gu,

Seoul 110-744, Korea.

Correspondence should be sent to Dr M. S. Park; e-mail: pmsmed@hanafos.com

(C)2006 British Editorial

Society of Bone and

Joint Surgery

doi:10.1302/0301-620X.88B2.

$16274 \$ 2.00$

$J$ Bone Joint Surg $[B r]$

2006;88-B:243-7.

Received 13 January 2005;

Accepted after revision

7 October 2005
Instability of the hip in cerebral palsy is commonly associated with dysplasia and or defects of the acetabulum. Previous authors have reported posterior, ${ }^{1,2}$ anterior, ${ }^{2-4}$ superior $^{2}$ and global (mixed) defects. ${ }^{2}$ Quantitative evaluation using transaxial CT has suggested that the defects in the wall are largely posterior. ${ }^{1}$ However, defects in the antero- and posterosuperior directions, which are important when considering pelvic reconstruction, were impossible to measure accurately. The development of threedimensional (3D) reconstruction ${ }^{5,6}$ has allowed the investigation and reporting of a greater variety of acetabular defects. ${ }^{2,7}$ However, conventional $3 \mathrm{D}$ reconstruction was inadequate for quantitative measurement. The advent of multidetector CT allowed thin sliced images to be retrieved easily so that multiplanar reformation and evaluation of volume became possible.

Our aim was to perform qualitative and quantitative evaluation of acetabular defects and also to compare the acetabular volume in patients with such defects with that of the normal acetabulum.

\section{Patients and Methods}

The study was covered by the institutional review board of Seoul National University Bundang Hospital.

We performed a retrospective study of 27 patients with cerebral palsy who had under- gone hip surgery since 2002. Medical records showed a total of 57 patients who had undergone pelvic osteotomy and/or femoral varus osteotomy for instability of the hip due to cerebral palsy during that period. Of these 57 patients, 27 ( 17 boys and ten girls) with available pre-operative 3D-CT data were selected. The 3D-CT data of the contralateral acetabula of ten patients with unilateral Perthes' disease were used as a control group. Of the study group, 19 patients had spastic quadriplegia, six spastic diplegia, and two mixed-type quadriplegia, with some component of dystonia. The mean age at the time of the 3D-CT evaluation was 7.2 years (SD 2.3) for the study group and 8.8 years (SD 2.3) for the control group. In one patient only, pre- and post-operative comparisons were performed since post-operative 3D-CT data were available.

CT scans (Mx8000IDT; Philips Medical Korea, Seoul, Korea) were acquired at a thickness of $1 \mathrm{~mm}$, from just above the anterosuperior iliac spine to just below the ischial tuberosity. We endeavoured to limit the dose of irradiation by using multidetector CT with age- and weight-specific paediatric protocols ${ }^{8}$ and confining the field of scan. The resultant dose was believed to be smaller than that of a conventional CT scan. Dicom-formatted files were retrieved; 3D reconstruction, multiplanar reformation, and volume integration were car- 


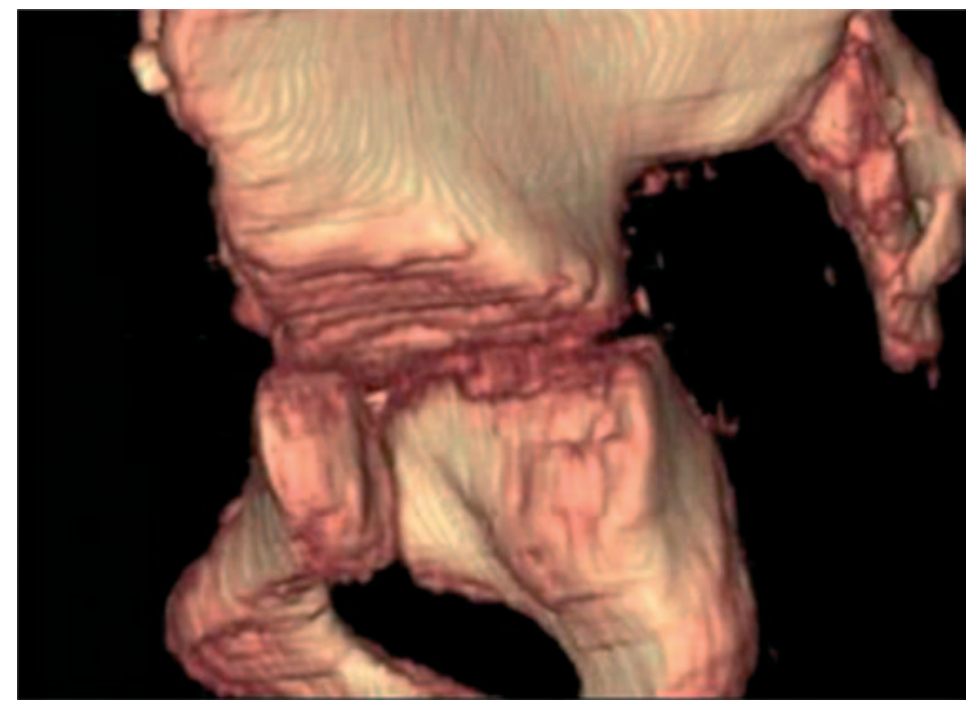

Fig. 1a

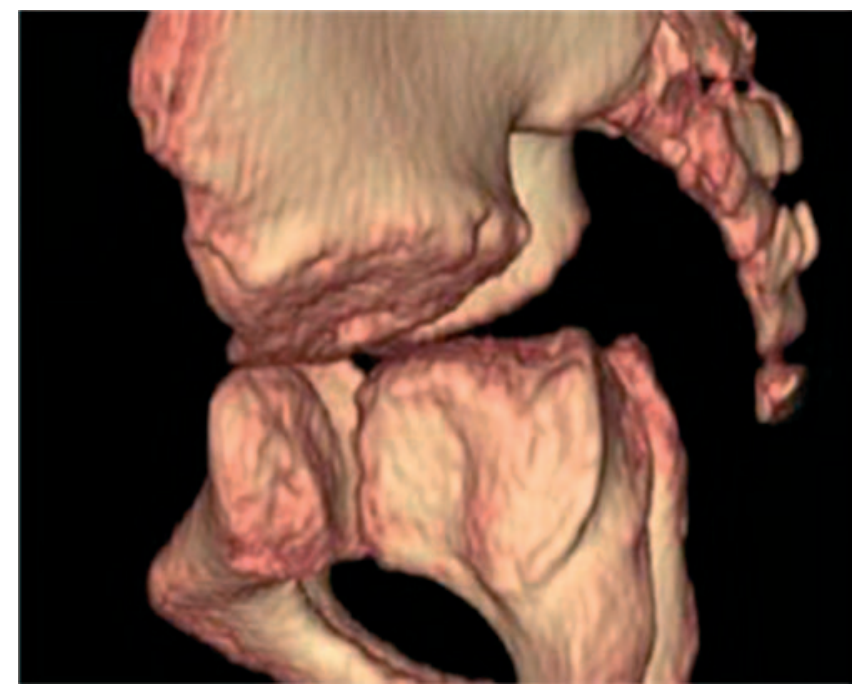

Fig. 1c

ried out using Rapidia software (version 2.8; Infinitt, Seoul, Korea).

Qualitative study. All hips were categorised into one of three groups, the subluxation group, the dislocation group, and the stable group, according to the 3D reconstructed lateral and tail-viewed images of the acetabulum and femoral head. The location of the acetabular defect was classified as either anterior, global (mixed), or posterior (Fig. 1), using the $3 \mathrm{D}$ reconstructed lateral images of the acetabulum. Three orthopaedic surgeons (CYC, MSP, KML) and a trainee (BW) independently examined the 3D reconstructed lateral images of the acetabulum without the femoral head allowing an assessment of internal intra-observer reliability to be made. The images were embedded in Powerpoint software (Microsoft, Redmond, Washington) and were displayed on a 19-inch identical computer monitor, with an identical setting for each examiner. Two examination ses-

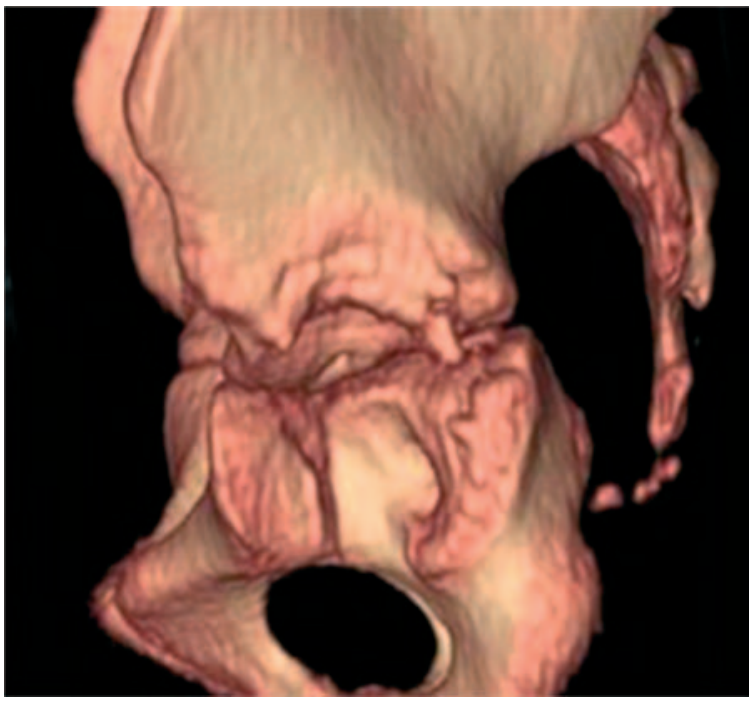

Fig. 1b

3D-CT scans of typical acetabula showing a) an anterior defect, b) a global defect and c) a posterior defect.

sions were conducted, with an interval of one month between the first and second. Images were randomly ordered by one of the authors, who did not participate in the sessions. Patient information was not given to any observer. A total of 54 acetabular images were examined. The orthopaedic surgeons had 20, five and four years experience, respectively and the trainee two years.

Quantitative study. Three-directional acetabular indices (anterosuperior, superolateral and posterosuperior) were designed and measured using multiplanar reformation (Fig. 2 ). The anterosuperior index was determined after oblique plane images had been obtained by performing multiplanar reformation, dissecting the anteroinferior iliac spine and the posterior end of the ischial tuberosity on the true lateral image. Subsequently, the angle between the horizontal line connecting the triradiate cartilages and that connecting the triradiate cartilage with the anterosuperior acetabular 


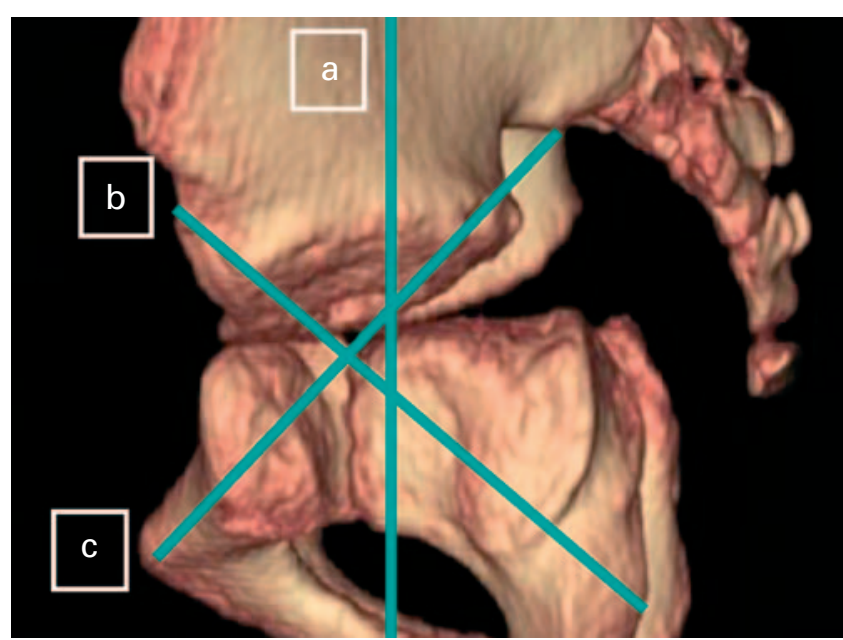

Fig. 2

CT scan showing measurement of the acetabular indices. Each line shows the cutting planes of a) the superolateral index, b) the anterosuperior index and c) the posterosuperior index.

margin were measured (Fig. 3). The superolateral index was measured in the same manner, in a plane which dissected the centre of the acetabulum parallel to the line connecting the anterosuperior iliac spine and the symphysis. The posterosuperior index was measured in the plane which dissected the line of the symphysis pubis and the posteroinferior iliac spine. The triradiate cartilage appeared consistently to pass through the median point of the acetabulum when measuring the anterosuperior or superolateral indices. However, in the posterosuperior index, both the inferior and posterior limbs of the triradiate cartilage were displayed and therefore we used the inferior limb as the co-ordinate for evaluation. Acetabular volume was also determined by multiplying the acetabular area in the axial image and the thickness of the slice (Fig. 4). For example, if the thickness of the slice was $1 \mathrm{~mm}$ and the measured acetabular area was 5 $\mathrm{mm}^{2}$, the volume of that slice of acetabulum was $5 \mathrm{~mm}^{3}$. If there were five slices with a volume of $3,4,5,4$ and $3 \mathrm{~mm}^{3}$, the total acetabular volume would be $19 \mathrm{~mm}^{3}$.

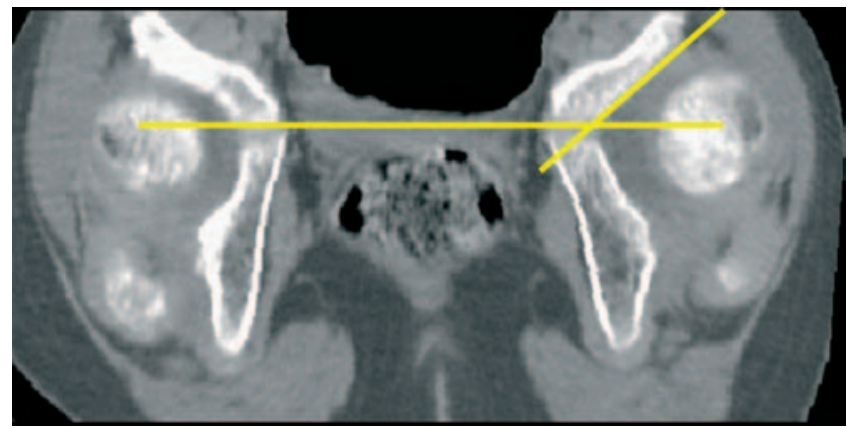

Fig. 3

Reformatted CT scans for the measurement of the anterosuperior index which was measured using a method similar to that used to determine the acetabular index.

Statistical analysis. This was performed using SPSS 11.0 (SPSS, Chicago, Illinois). Comparisons between the study and control groups in the quantitative assessment were made using Wilcoxon rank-sum tests, and kappa statistics were used to assess intra- and interobserver reliability in the qualitative group. ${ }^{9}$ The classic interpretation of kappa statistics is as follows: 0.00 to 0.20 , poor; 0.21 to 0.40 , fair; 0.41 to 0.60 , moderate; 0.61 to 0.80 , substantial and 0.81 to 1.00 , almost perfect agreement. ${ }^{9}$ We considered $\mathrm{p}$ values of 0.05 or less to be significant.

\section{Results}

Qualitative study. Qualitative analysis of the location of the defects is shown in Figure 1 and Table I. No statistically significant differences in the mean age were seen for the dislocation, subluxation and control groups. However, the mean age of the study group was 1.6 years less than that of the control group. Posterior defects predominated in the subluxation group and global defects in the dislocation group (Table I).

Intra-observer reliability varied from 0.50 to 0.81 in terms of kappa statistics (Table II), which implies moderate to substantial agreement. ${ }^{9}$ The mean interobserver reliability also showed substantial agreement (Table III).

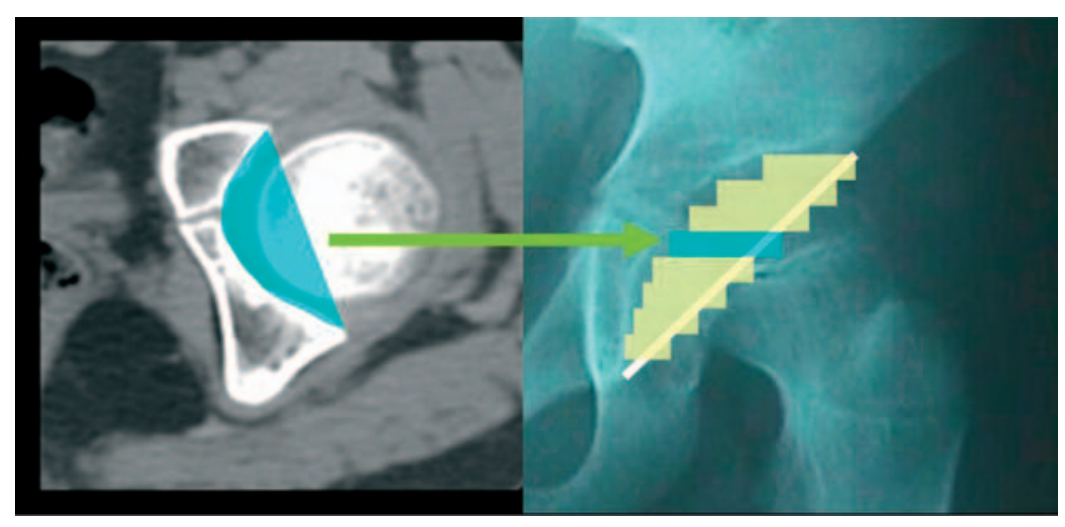

Fig. 4

CT scans showing that considering the thickness of the plane, the acetabular volume was calculated by integration of the area of the acetabulum. 
Table I. Qualitative analysis of the acetabular defects, by number and percentage

\begin{tabular}{llccc}
\hline & Anterior & Global & Posterior & Minimal \\
\hline Dislocation $(n=29)$ & $1(3)$ & $23(80)$ & $5(17)$ & - \\
Subluxation $(n=20)$ & $2(10)$ & $3(15)$ & $15(75)$ & - \\
Stable $(n=5)$ & - & - & $3(60)$ & $2(40)$ \\
Total $(n=54)$ & $3(6)$ & $26(48)$ & $23(43)$ & $2(4)$ \\
\hline
\end{tabular}

Table II. Intra-observer reliability between the first and second sessions

\begin{tabular}{lllll}
\hline Rater & Experience & Kappa statistic & $\%$ of agreement & Reliability \\
\hline $1^{\text {st }}$ & 20 years & 0.81 & 89 & Almost perfect \\
$2^{\text {nd }}$ & 5 years & 0.77 & 88 & Substantial \\
$3^{\text {rd }}$ & 4 years & 0.79 & 89 & Substantial \\
$4^{\text {th }}$ & Trainee & 0.50 & 74 & Moderate \\
\hline
\end{tabular}

Table III. Inter-observer reliability (mean values)

\begin{tabular}{llll}
\hline Session & Kappa statistic & $\%$ of agreement & Reliability \\
\hline $1^{\text {st }}$ & 0.62 & 79 & Substantial \\
$2^{\text {nd }}$ & 0.60 & 79 & Substantial \\
Overall & 0.61 & 79 & Substantial \\
\hline
\end{tabular}

Table IV. Acetabular indices (mean $\pm \mathrm{SD} ;^{\circ}$ ) according to the acetabular defects (AS, anteroposterior; SL, superolateral; PS, posterosuperior)

\begin{tabular}{llll}
\hline & AS index & SL index & PS index \\
\hline Global & $38 \pm 10^{*}$ & $41 \pm 10^{*}$ & $58 \pm 8^{*}$ \\
Posterior & $33 \pm 9^{*}$ & $32 \pm 9^{*}$ & $56 \pm 9^{*}$ \\
Anterior & $38 \pm 7^{*}$ & $34 \pm 11^{*}$ & $56 \pm 9^{*}$ \\
Control & $20 \pm 5$ & $17 \pm 3$ & $39 \pm 3$ \\
\hline
\end{tabular}

* significant compared with control group

Table V. Comparison of the acetabular indices (mean $\pm \mathrm{SD} ;{ }^{\circ}$ ) and volume in the three groups (AS, anteroposterior; SL, superolateral; PS, posterosuperior)

\begin{tabular}{lllllc}
\hline & Age (yrs) & AS index & SL index & PS index & Volume (m) \\
\hline Dislocation & $7.4 \pm 2.5$ & $37 \pm 10$ & $40 \pm 10$ & $58 \pm 8$ & $3.4 \pm 2.0$ \\
Subluxation & $6.4 \pm 1.6$ & $33 \pm 8$ & $30 \pm 9$ & $54 \pm 10$ & $5.2 \pm 2.8$ \\
Control & $8.8 \pm 2.3$ & $20 \pm 5$ & $17 \pm 3$ & $39 \pm 3$ & $14.2 \pm 6.0$ \\
\hline
\end{tabular}

Quantitative study. In patients with any type of acetabular defect, all indices were significantly higher than those of the control group, regardless of the site of the defect $(\mathrm{p}=0.004)$. The superolateral index of the global defect group was significantly higher $(\mathrm{p}=0.004)$ than that of the posterior defect group (Table IV).

When instability of the hip was considered in the dislocation and subluxation groups, both groups showed a significant increase $(\mathrm{p}<0.001)$ in all three indices compared with control values. Furthermore, anterosuperior, superolateral and posterosuperior indices were consistently higher in the dislocation group than in the subluxation group but only for the superolateral index was this statistically significant $(\mathrm{p}=0.001$; Table V).

The mean acetabular volume in the dislocated hips was significantly lower than that of the subluxated hips ( $\mathrm{p}=$ 0.007 ) and the mean acetabular volume of the subluxated hips was significantly lower than that of the control group $(\mathrm{p}<0.001 ;$ Table V).

\section{Discussion}

Instability of the hip (subluxation or dislocation) has been reported to occur in about $21 \%$ of patients with cerebral palsy, ${ }^{10}$ and $50 \%$ of quadriplegic patients. ${ }^{11}$ Severe dislocation is associated with acetabular defects (dysplasia). ${ }^{6}$ When the migration index is $50 \%$ to $70 \%$, pelvic osteotomy is recommended and has been reported to be associated with a better prognosis. ${ }^{12,13}$ Early attempts were made to determine the shape and location of the acetabular defects by simple radiography or conventional CT. ${ }^{1,3}$ How- ever, since the former projects the coronal plane and conventional CT the transverse sectional image, the $3 \mathrm{D}$ features of the acetabulum cannot be accurately visualised. The advent of 3D-CT, has made the evaluation of acetabular defects practicable. ${ }^{2}$

The reliability of qualitative analysis has yet to be demonstrated. The accurate measurement of acetabular defects by 3D-CT can be quantified by using a hypothetical factor, such as 'the acetabular index' on plain anteroposterior radiographs of the hip. Therefore, the multiplanar reformation technique was used to determine 'the acetabular index' for the anterosuperior, superolateral and posterosuperior wall of the acetabulum. In both the dislocation and subluxation groups, all three indices were found to be higher than in the control group, indicating that the acetabula in patients with cerebral palsy with instability of the hip were shallower than those of the control group. Therefore, it is possible that posterior or anterior defects seen on qualitative assessments were actually global defects although intra- and interobserver reliability was moderate to substantial. Therefore, it may be desirable to use quantitative indices, as well as gross visual analysis when evaluating acetabular defects.

The pathomechanics of dislocation in cerebral palsy is not yet fully understood. Some authors have suggested a hypothesis relating the forces around the joint to the direction of dislocation and the location of the defect. ${ }^{2,14} \mathrm{~A}$ peculiar phenomenon noted in our study was that, as subluxation progressed to dislocation, posterior acetabular defects progressed to global defects, and the superolateral 
index in patients with dislocation was higher than that in the subluxation group. Furthermore, volume decreased in the dislocation group. It is possible that the superolateral wall defects and decreased volume may constitute a sine qua non of dislocation, and that an osteotomy which increases acetabular volume and covers the defects, particularly the superolateral defect, is necessary for the maintenance of the reduction.

Quantitative analysis including the determination of indices and volumes may be useful in the assessment of the outcome of pelvic osteotomy. However, post-operative CT scans were only available in one patient in whom the volume actually increased after the modified Dega osteotomy (mean $3.5 \mathrm{ml}$ increased to $7.3 \mathrm{ml}$ ).

No study has yet attempted to evaluate acetabular volume and a belief that a Dega or Pemberton pelvic osteotomy reduces the acetabular volume still holds sway. However, it is clear that a 'cup' contains more water than a 'dish' with an identical surface area and in our one case with post-operative CT evaluation osteotomy increased the acetabular volume.

There are some limitations inherent in our study. It was retrospective and the mean age of the study group was 1.6 years less than that of the control group. This may be important since patients with severe cerebral palsy often shown retardation of bone age compared with chronological age and acetabular volume may be correlated with age. However, the volume of the acetabulum in the subluxation group was greater than that in the dislocation group, even though the age of the subluxation group was less than that of the dislocation group. The triradiate cartilage and acetabular bone margin were used principally as co-ordinates in the determination of the indices. The thickness of the triradiate cartilage may also be influenced by agedependent ossification. Therefore, changes associated with age and ossification should be considered when interpreting our results.

In this study subluxation was associated primarily with a posterior defect. However, as it progressed to dislocation, global defects became more prominent. Acetabular volume also gradually decreased from the control group to the subluxation and dislocation groups. Quantitative methods, including measurement of anterosuperior, superolateral and posterosuperior indices, should be considered since dependence solely on qualitative analysis may lead to error.

\section{Supplementary Material}

$\ddot{e}$ A table with patient details is available with the electronic version of this article on our website at www.jbjs.org.uk

No benefits in any form have been received or will be received from a commercial party related directly or indirectly to the subject of this article.

\section{References}

1. Buckley SL, Sponseller PD, Magid D. The acetabulum in congenital and neuromuscular hip instability. J Pediatr Orthop 1991;11:498-501.

2. Kim HT, Wenger DR. Location of acetabular deficiency and associated hip dislocation in neuromuscular hip dysplasia: three-dimensional computed tomographic analysis. J Pediatr Orthop 1997;17:143-51.

3. Gugenheim JJ, Gerson LP, Sadler C, Tullos HS. Pathologic morphology of the acetabulum in paralytic and congenital hip instability. J Pediatr Orthop 1982;2:397-400.

4. Pope DF, Bueff HU, DeLuca PA. Pelvic osteotomies for subluxation of the hip in cerebral palsy. J Pediatr Orthop 1994;14:724-30.

5. Abel ME, Sutherland DH, Wenger DR, Mubarak SJ. Evaluation of CT scans and 3-D reformatted images for quantitative assessment of the hip. J Pediatr Orthop 1994; 14:48-53.

6. Abel MF, Wenger DR, Mubarak SJ, Sutherland DH. Quantitative analysis of hip dysplasia in cerebral palsy: a study of radiographs and 3-D reformatted images. J Pediatr Orthop 1994;14:283-9.

7. Brunner R, Picard C, Robb J. Morphology of the acetabulum in hip dislocations caused by cerebral palsy. J Pediatr Orthop B 1997;6:207-11

8. Hugh TM. Dose reduction for CT pediatric imaging. Pediatr Radiol 2002;32:724-8.

9. Landis JR, Koch GG. The measurement of observer agreement for categorical data. Biometrics 1977:33:159-74.

10. Renshaw TS, Green NE, Griffin PP, Root L. Cerebral palsy: orthopaedic management. Instr Course Lect 1996;45:475-90.

11. Gamble JG, Rinsky LA, Bleck EE. Established hip dislocations in children with cerebral palsy. Clin Orthop 1990;253:90-9.

12. Noonan KJ, Walker TL, Kayes KJ, Feinberg J. Varus derotation osteotomy for the treatment of hip subluxation and dislocation in cerebral palsy: statistical analysis in 73 hips. J Pediatr Orthop B 2001;10:279-86.

13. Song HR, Carroll NC. Femoral varus derotation osteotomy with or without acetabuloplasty for unstable hips in cerebral palsy. J Pediatr Orthop 1998;18:62-8.

14. Miller F, Slomczykowski M, Cope R, Lipton GE. Computer modeling of the pathomechanics of spastic hip dislocation in children. J Pediatr Orthop 1999;19:486-92. 